\title{
Biomechanical Analysis of the Throwing Athlete and Its Impact on Return to Sport
}

\author{
Nicholas A. Trasolini, M.D., Kristen F. Nicholson, Ph.D., Joseph Mylott, B.S., \\ Garrett S. Bullock, P.T., D.P.T., D.Phil., Tessa C. Hulburt, M.S., and \\ Brian R. Waterman, M.D.
}

\begin{abstract}
Throwing sports remain a popular pastime and frequent source of musculoskeletal injuries, particularly those involving the shoulder and elbow. Biomechanical analyses of throwing athletes have identified pathomechanic factors that predispose throwers to injury or poor performance. These factors, or key performance indicators, are an ongoing topic of research, with the goals of improved injury prediction, prevention, and rehabilitation. Important key performance indicators in the literature to date include shoulder and elbow torque, shoulder rotation, kinetic chain function (as measured by trunk rotation timing and hip-shoulder separation), and lower-extremity mechanics (including stride characteristics). The current gold standard for biomechanical analysis of the throwing athlete involves marker-based 3dimensional) video motion capture. Emerging technologies such as marker-less motion capture, wearable technology, and machine learning have the potential to further refine our understanding. This review will discuss the biomechanics of throwing, with particular attention to baseball pitching, while also delineating methods of modern throwing analysis, implications for clinical orthopaedic practice, and future areas of research interest. Level of Evidence: V, expert opinion.
\end{abstract}

$\mathbf{T}$ hrowing sports remain a popular pastime and frequent source of musculoskeletal injuries, particularly those involving the shoulder and elbow. ${ }^{1-3}$ The biomechanics of throwing and their impact on injury risk have been extensively studied dating back to the 1980s. ${ }^{4-7}$ Early work in understanding the pathomechanics of throwing injuries relied on detailed video analysis, ${ }^{4}$ radar, ${ }^{8}$ and electromyography. ${ }^{5,6}$ Although increased sophistication of 3-dimensional (3D) motion capture and complex data analysis have engendered a

From the Department of Orthopaedic Surgery, Wake Forest School of Medicine, Winston-Salem, North Carolina, U.S.A.

The authors report the following potential conflicts of interest or sources of funding: N.A.T. reports other from DJO, outside the submitted work. B.R.W. reports other from American Academy of Orthopaedic Surgeons, American Orthopaedic Society for Sports Medicine, Arthrex, Arthroscopy, Elsevier, Kaliber AI, Sparta Science, Vericel, and Vivorte, outside the submitted work. Full ICMJE author disclosure forms are available for this article online, as supplementary material.

Received September 13,2021; accepted September 30, 2021.

Address correspondence to Nicholas A. Trasolini, M.D., Department of Orthopaedic Surgery, Atrium Health Wake Forest Baptist, 1 Medical Center Blvd., Winston-Salem, NC 27157. E-mail: TrasoliniMD@gmail.com

(C) 2021 THE AUTHORS. Published by Elsevier Inc. on behalf of the Arthroscopy Association of North America. This is an open access article under the CC BY-NC-ND license (http://creativecommons.org/licenses/by-nc-nd/4.0/).

2666-061X/211318

https://doi.org/10.1016/j.asmr.2021.09.027 more nuanced understanding of throwing mechanics, the fundamental principles have remained unchanged. These principles include the phases of throwing, ${ }^{7} \mathrm{ki}-$ netic chain, ${ }^{9}$ upper-extremity biomechanics, ${ }^{5,6,10}$ and throwing pathomechanics. ${ }^{8}$ Understanding these foundational concepts is a prerequisite for understanding modern throwing analysis. This review will discuss the biomechanics of throwing, with particular attention to baseball pitching, while also delineating methods of modern throwing analysis, implications for clinical orthopaedic practice, and future areas of research interest.

\section{Biomechanics of Throwing}

\section{Phases of Throwing}

Throwing a baseball is a complex movement that requires coordination of many steps in a specific sequence to maximize performance and reduce injury risk. The phases of throwing (Fig 1) include wind-up, stride, arm cocking, arm acceleration, arm deceleration, and follow through. ${ }^{7,9,11}$ Each phase creates or transfers energy through the body to the arm and to the baseball. While originally described for baseball pitching, these phases are largely consistent for football throwing as well. ${ }^{12}$ 


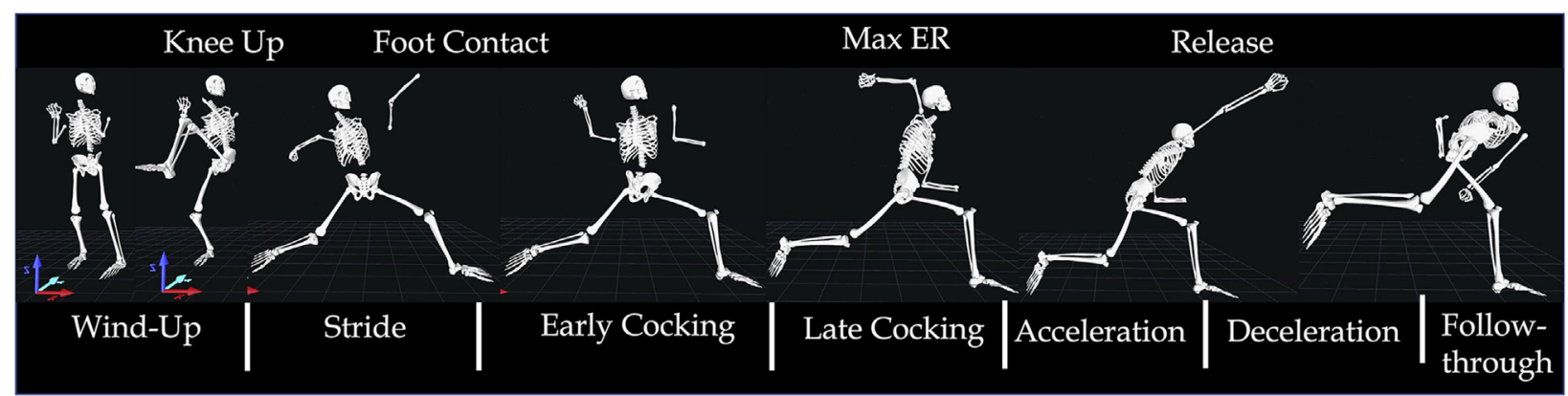

Fig 1. The phases of pitching (as described by Fleisig et al. ${ }^{8}$ ) demonstrated with skeletal diagrams created during video throwing analysis. (ER, external rotation.)

The throw begins with the wind-up phase, where weight is transferred to the drive leg and potential energy is stored in the form of drive knee bending and truncal rotation. The drive leg exhibits a hip hinging pattern to activate the posterior chain. ${ }^{11}$ The stride leg (or lead leg) is lifted to shift the center of mass over the drive leg. This phase ends when the knee lift reaches its peak and center of mass begins to shift toward the stride leg. ${ }^{11}$

The stride phase includes all motion from the maximum lead knee lift until foot strike. Early in this phase, the athlete maintains their hip hinge with the drive leg but pushes into the ground generate a ground reaction force that will allow them to move linearly toward their target (Fig 2). The length of the stride has been shown to correlate with both velocity ${ }^{13}$ and elbow varus torque, ${ }^{14}$ but a stride length of greater than $80 \%$ body height may protect against those elbow torque increases. ${ }^{15}$ Stride location is also important; the foot should land in line with the drive leg with the foot pointed slightly internal. ${ }^{16}$ As the lead leg gets closer to the ground, the pelvis begins to rotate independently of the torso, creating what is commonly known as hip-shoulder separation. By the completion of the stride phase, the throwing arm should have around $90^{\circ}$ shoulder abduction, $>35^{\circ}$ shoulder external rotation, and $>90^{\circ}$ elbow flexion to minimize risk of injury. ${ }^{17,18}$

The arm cocking phase begins at foot strike and ends at maximum shoulder external rotation (MER). The lead leg serves as a stable base for rotation for the remainder of the throw, so lead knee flexion should be at its greatest at foot strike and decrease before ball release. Soon after foot strike the pelvis should stop rotating, allowing for energy transfer up to the torso. Rotation of the upper half will close the hip-shoulder separation previously created and allow for the throwing arm to externally rotate even more. Ideally, the shoulder will reach $170^{\circ}$ to $180^{\circ}$ of MER while maintaining $90^{\circ}$ shoulder abduction and $>90^{\circ}$ elbow flexion. ${ }^{9}$ Limitations of external rotation can reduce ball velocity ${ }^{19}$ and increase shoulder joint loading. ${ }^{20}$
Once MER has been achieved, the arm begins to accelerate toward the throwing target. This phase, appropriately named the arm-acceleration phase, consists of the time between MER and ball release. While the arm moves towards the target, the torso continues to rotate and tilt forward until stopping rotation just before ball release, generating and transferring as much energy as possible to the arm. During this time, the lead knee is extending to better stop the pelvis and transfer energy from the lower extremities to the torso to the arm. The shoulder switches from externally rotating to internally rotating and the elbow extends to transfer energy to the hand and baseball at ball release. This phase creates the highest demands of force and torque on the shoulder and elbow with peak angular velocities for professional pitchers of $\sim 6,200 \mathrm{deg} / \mathrm{s}$ and $\sim 4,600$ $\mathrm{deg} / \mathrm{s}$ for shoulder internal rotation and elbow extension, respectively. ${ }^{21}$

Following ball release, the arm deceleration phase continues until maximum shoulder internal rotation. Now that the athlete's arm has reached this extremely high angular velocity and released the ball, the arm must decelerate in a safe, controlled manner. After ball release, the torso rotates and tilts forward again to clear space for the arm as the forearm continues to pronate as well. The muscles of the shoulder, arm, upper back, and chest work under high stress to slow down the arm and decrease joint loading.

The follow-through phase consists of any movement after the shoulder reaches maximum shoulder internal rotation until the arm is no longer in motion. This phase can look very different for each athlete depending on their arm slot, lower half mechanics, and other factors.

\section{Kinetic Chain}

Throwing athletes generate velocity through a synchronized transfer of core and lower-extremity energy to upper-extremity torque, rotation, and angular velocity. Potential energy stored during the wind-up through weight transfer and truncal rotation loads the body like a torsion spring. This energy is then 


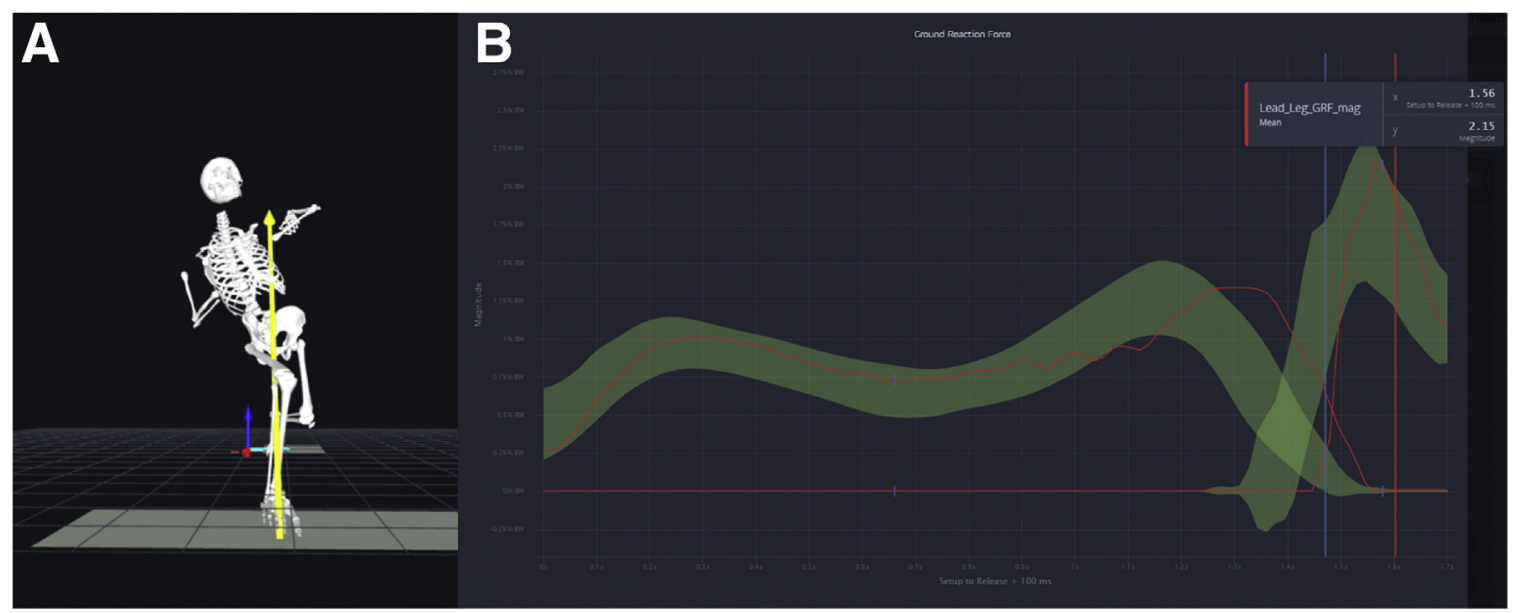

Fig 2. Example of ground reaction force measurement during throwing analysis. (A) Skeletal model based on 3-dimensional marker data. The yellow vector represents the resultant ground reaction force measured with force plates embedded in the pitching mound. (B) Representative graph of the plant leg and lead leg ground reaction force. The green area represents the mean for college pitchers.

transferred to the shoulder and elbow and converted to kinetic energy and centripetal force that accelerates the ball in the desired direction. Efficient summation and transfer of core and lower-extremity potential energy to upper extremity kinetic energy is critical for high-level performance and injury prevention. The mechanism that facilitates that energy transfer has been termed the kinetic chain. ${ }^{22}$

A functional kinetic chain consists of 3 components: optimized anatomy, sequential generation of forces, and efficient motor patterns. ${ }^{23}$ Optimized anatomy refers to strength, flexibility, and power of the many independent functional segments of the body, or kinetic links. ${ }^{24}$ Important kinetic links for throwing include the feet, lower extremities, hip and pelvis, trunk, scapulothoracic articulation, shoulder and elbow, and distal extremity. ${ }^{25}$ During a throw, forces are sequentially generated by the various segments of the kinetic chain and coordinated to accelerate the ball in the desired direction. Efficient task-specific motor patterns allow for minimal energy loss during transfer between independent segments of the kinetic chain.

Dysfunction of the kinetic chain can occur due to disruption of anatomy (e.g., loss of shoulder range of motion, lack of hip internal rotation), inappropriate distribution of forces between segments (relying too much on arm strength without lower-extremity activation), or inefficient motor patterns (scapular dyskinesis). ${ }^{22}$ It has been reported that the legs and trunk account for $51 \%$ to $55 \%$ of the kinetic energy delivered to the hand during a throw. ${ }^{26}$ When the kinetic chain is not functioning correctly, the upper extremity tries to "catch up," which increases the forces on the shoulder and elbow placing players at risk for injury. ${ }^{27}$ This was highlighted by a recent study comparing professional pitchers with upper-extremity injuries with those who completed the season without injury. ${ }^{28}$ During both stride leg and drive leg balance tasks, injured pitchers were found to have significantly worse lumbopelvic control consistent with a dysfunctional kinetic chain.

Evaluation of kinetic chain dysfunction can be performed with a stepwise proximal to distal approach, which has been described by Kibler et al. and others. ${ }^{22,26}$ Each link in the kinetic chain is separately evaluated for strength, range of motion, coordination, and internal derangements. Preventative training or postinjury rehabilitation can be tailored to address dysfunctional links in the kinetic chain and improve coordination between segments. ${ }^{25}$ During modern throwing analysis, kinetic links are examined before the throwing task. Video motion capture is then used to examine the efficiency of the kinetic chain. Hip-shoulder separation (Fig 3) is a key indicator of this kinetic chain function, as it represents the loading of lower extremity into a torsion spring through the core. It has been demonstrated that hip-shoulder separation at front foot contact correlates with trunk rotation velocity, which in turn correlates with pitch velocity. ${ }^{13,29}$

\section{Shoulder and Elbow Biomechanics}

The upper extremity becomes active starting in the stride phase of throwing with initial external rotation and abduction of the humerus into a semicocked position. ${ }^{16,27}$ With 6 degrees of freedom, the scapula protracts, tilts anteriorly, and rotates laterally. ${ }^{11}$ The trunk and shoulder translate toward the target as the stride ends, but the elbow and hand lag behind resulting in extreme shoulder external rotation. Scapular position changes to accommodate this motion within the subacromial space. At maximum external rotation, the scapula is positioned in maximum retraction, lateral 


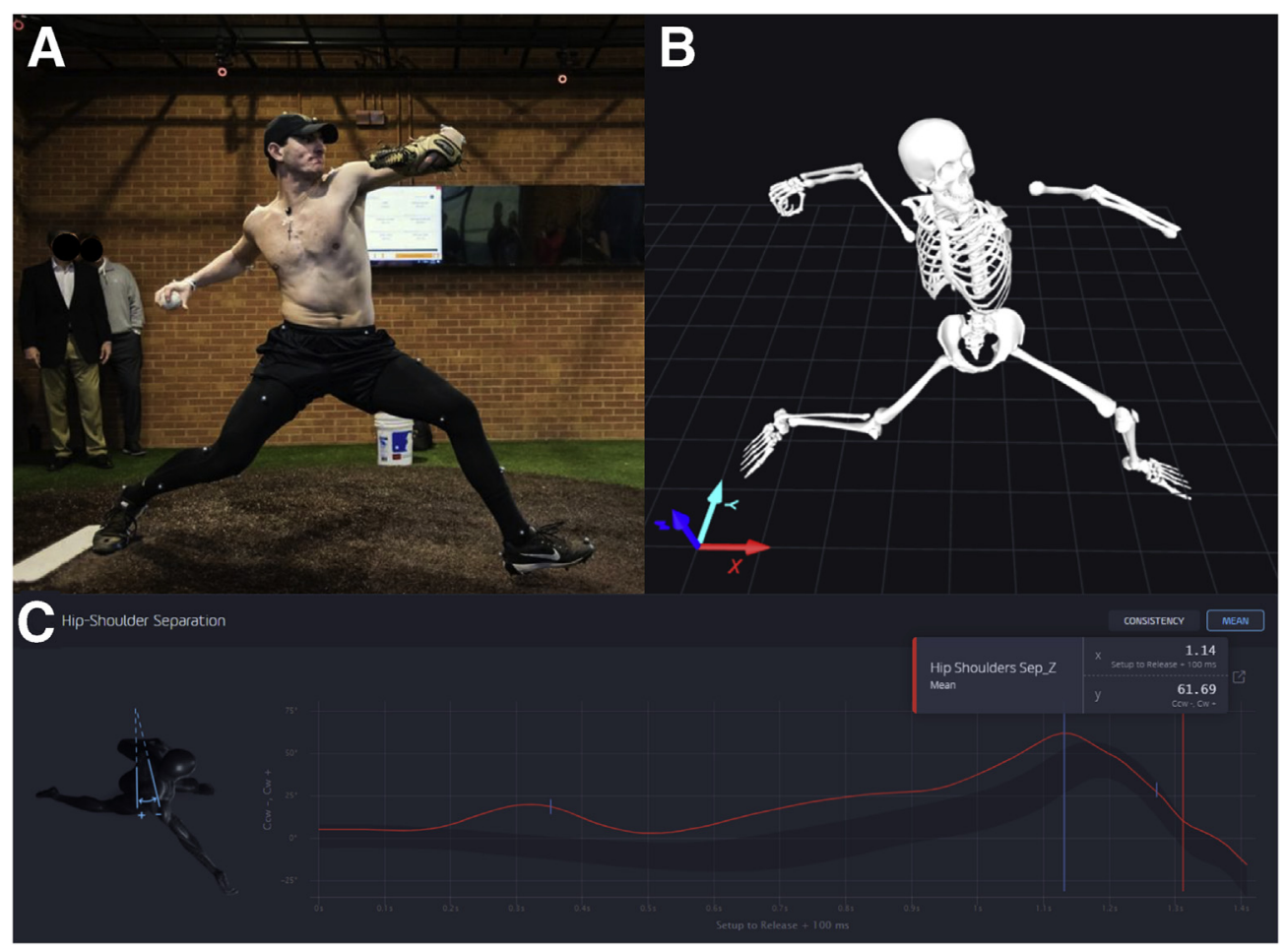

Fig 3. Example of a 3dimensional (3D) biomechanical throwing analysis. (A) Right-handed pitcher wearing a 41 retroreflective marker set. (B) Skeletal model based on 3D marker data. (C) A graph of hip-shoulder separation, a measure of the rotational difference between the pelvis and the trunk, and just one of the kinematic metrics calculated in a 3D biomechanical analysis.

rotation, and posterior tilt. ${ }^{11}$ The elbow flexes and the hand is maintained on top of the ball. The rotator cuff activates in concavity compression to maintain the stability of the glenohumeral joint." ${ }^{5,27}$ A "critical instant" occurs just before MER and the maximum elbow varus torque is reached. ${ }^{8}$

As the throw progresses from late cocking to early acceleration, there is a large transfer of potential energy to kinetic energy, which places a great deal of stress on the glenohumeral and elbow joints. In the shoulder, shear forces on the anterior and superior aspect of the joint must be resisted by the capsule, rotator cuff, and labrum. ${ }^{26}$ In the elbow, a supraphysiologic valgus load is placed on the ulnar collateral ligament, which is partially shielded by the flexor pronator mass, biceps, and triceps. ${ }^{26}$ At this stage, elbow flexion angle determines the perpendicular distance between the ball and the long axis of the humerus thereby controlling the axial torque on the humerus and glenohumeral joint. $^{16,26}$ Elbow extension begins slightly before

Table 1. Steps in a Pitching Evaluation at Our Institution

1. OnBaseU* functional movement screen

2. Assessment for scapular dyskinesis

3. Shoulder and hip passive range of motion (ROM)

4. Rotator cuff and grip strength

5. Player selected warm-up

6. 3D biomechanical evaluation (12 motion analysis cameras: surrounding the mound at approximately 12 feet; 4 high-speed video cameras: front, back, overhead, open side; 3 multi-axial force plates: 1 under the rubber, 2 in the "landing" zone)

a) Place 41 retroreflective markers (Fig 4)

b) Static standing trial

c) Acclimation pitches without data collection

d) Four pitches of each pitch type in the player's repertoire (4-seam fastball, sinker, slider, curveball, change up, etc.) with motion analysis, video, and force collected simultaneously

e) Removal of markers

7. Calculations, feedback, and intervention

a) Full body kinematics, kinetics, and spaciotemporal variables are calculated (Table 2 lists some key performance indicators)

b) Web-based reports are generated

c) Biomechanical, ROM, strength, and mobility feedback added to reports

d) Follow up session to discuss correctives based on feedback

*OnBaseU (Oceanside, CA). 


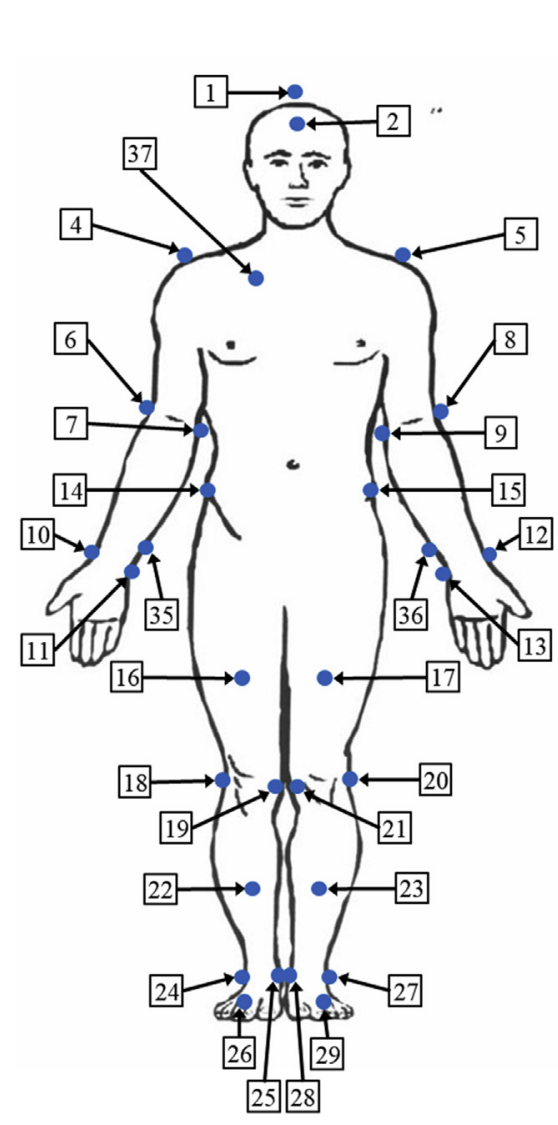

1. Top of head

2. Anterior head

3. Posterior head

4. Right acromion

5. Left acromion

6. Right lateral epicondyle of the humerus

7. Right medial epicondyle of the humerus

8. Left lateral epicondyle of the humerus

9. Left medial epicondyle of the humerus

10. Right radial styloid

11.Right ulnar styloid

12.Left radial styloid

13.Left ulnar styloid

14. Right anterior superior iliac spine (ASIS)

15.Left ASIS

16.Right anterior thigh

17.Left anterior thigh

18. Right lateral femoral epicondyle

19. Right medial femoral epicondyle

20.Left lateral femoral epicondyle

21.Left medial femoral epicondyle

22. Right anterior shank

23.Left anterior shank

24. Right lateral malleolus

25. Right medial malleolus

26. Right toe - between second and third metatarsals

27.Left lateral malleolus

28.Left medial malleolus

29. Left toe - between second and third metatarsals

30. Sacrum

31.Left heel - superior-posterior calcaneal tuberosity

32. Right heel - superior-posterior calcaneal tuberosity

33. Left dorsal hand

34. Right dorsal hand

35. Right medial forearm

36.Left medial forearm

37. Right clavicle

38.Left scapula medial supraspinous fossa

39. Right scapula medial supraspinous fossa

40.Left scapula posteriomedial border

41. Right scapula posteriomedial border

Fig 4. Marker locations for video motion capture throwing analysis.

humeral internal rotation to reduce the moment of inertia of the upper extremity kinetic link and allow for greater angular velocity with less torque. ${ }^{16}$

The completion of the throw involves humeral internal rotation and elbow extension as the ball accelerates towards the target. Once the ball is released, the arm experiences a second "critical instant" where the maximum glenohumeral distraction force occurs. ${ }^{8}$ The shoulder must be safely decelerated, compressed, and stabilized by forceful contraction of the infraspinatus, teres minor and major, latissimus dorsi, and posterior deltoid. ${ }^{11}$ Elbow extension must also be decelerated by way of eccentric contraction of the elbow flexors. The scapula decelerates and derotates back to its resting position through action of the trapezius, rhomboids, and serratus anterior. ${ }^{30}$ Finally, the follow through dissipates the remaining kinetic energy of the throw through the stride leg as the arm reaches its terminal position of internal rotation and adduction.

\section{Modern Throwing Analysis}

Modern throwing analysis is designed to capture a wide breadth of the biomechanical variables discussed previously from a series of recorded throws. These variables are referred to as key performance indicators (KPIs) and are compared with population norms throughout the phases of throwing for a given level of competition (i.e., youth, high school, collegiate, or professional). The steps for a complete video motion capture pitching assessment at our institution are outlined in Table 1 (Fig 4).

In brief, players present initially for a physical examination and functional movement evaluation. This is followed by a warm-up, which is left to player preference. Motion-capture markers are then applied followed by acclimation of video motion capture equipment. Finally, players will throw a series of pitches that are subsequently analyzed for KPIs, which are outlined in Table 2. Once this analysis is performed, a follow-up session is scheduled to discuss the results and educate the player on biomechanical factors that may increase their risk for poor performance or injury. While it has been used in prior throwing analysis research, $5,6,10,31,32$ electromyography is not routinely performed at our institution.

\section{Pathomechanics}

From a clinical standpoint, there are 2 key questions regarding throwing analysis: (1) Can biomechanical 
Table 2. Key Performance Indicators (KPIs) Measured at Our Institution

\begin{tabular}{ll}
\hline Ball Speed & Shoulder Abduction/horizontal Abduction at Foot Strike \\
Stride length & Shoulder abduction at release \\
Back leg GRF max & Maximum shoulder external rotation (MER) \\
Lead leg GRF max & Elbow angle at MER \\
Pelvis rotation at MER & Elbow angle at release \\
Hip-shoulder separation at foot strike & Elbow angle at foot strike \\
Trunk lateral tilt at foot strike & Max elbow varus torque \\
Trunk forward flexion at foot strike & Thorax angular velocity max \\
Knee flexion angle at release & Humerus angular velocity max \\
Trunk forward flexion at release & Elbow extension velocity max \\
Trunk lateral tilt at release & Pelvis angular velocity max \\
Trunk rotation at release & Lead knee angular velocity max \\
Time between max pelvis rotation velocity and max trunk rotation velocity &
\end{tabular}

NOTE. Bolded font indicates the most clinically relevant KPIs.

GRF, ground reaction force; MER, maximum shoulder external rotation.

measurements (KPIs) predict injury, and (2) can we modify abnormal mechanics to prevent injury? There has been extensive research investigating musculoskeletal health in baseball, but relatively few papers that directly address these questions. In a scoping review of 583 articles relating to musculoskeletal health and baseball, only 24 studies ( $5 \%$ ) compared attributes of injured and uninjured players, and only 11 studies $(2 \%)$ were identified that directly investigated injury prevention. ${ }^{1}$ Nevertheless, some throwing parameters have been identified in the literature as risk factors for injury. Chalmers et al. ${ }^{33}$ reviewed the relationship between throwing mechanics and injury and noted that the most significant factors included elbow varus torque, elbow flexion at ball release, altered knee flexion at ball release, fatigue, shoulder external rotation torque, and early trunk rotation (i.e., loss of hip-shoulder separation).

Elbow varus torque has been extensively studied. Elbow varus torque is correlated with pitch velocity, MER, and arm slot at ball release. ${ }^{34}$ Relationships have been reported between elbow varus torque and increased risk of elbow injuries. In a study of 23 professional pitchers monitored for 3 years after throwing analysis, those with increased shoulder and elbow torque values had higher rates of elbow injury. ${ }^{35}$

Early trunk rotation and loss of hip-shoulder separation are other pathologic KPIs that are measured during throwing analysis. As discussed, these factors are measured to evaluate the thrower for kinetic chain dysfunction. Improper trunk rotation sequences have been shown to increase shoulder forces by $9.2 \%$ body weight. $^{36}$

While most throwing analyses occur during a short pitching session with few pitches, this may fail to identify pathomechanics that develop with fatigue. In a study of 28 adolescent pitchers, Erickson et al. ${ }^{37}$ identified deterioration of kinetic chain mechanics as pitch count increased during a simulated game. Specifically, hip-shoulder separation decreased while upperextremity variables remained unchanged. Hip-shoulder separation is critical for energy transfer through the core muscles. ${ }^{9}$ With less efficient core muscle energy transfer, there is increased demand on the throwers shoulder and elbow if the same energy at ball release is to be achieved. ${ }^{33}$ Consequently, pitchers in this study lost velocity and had increased reports of arm pain as pitch count increased. In another simulated game study of 11 pitchers, the impact of fatigue on elbow varus torque was examined specifically. ${ }^{38}$ After the third inning, medial elbow torque began to increase by $0.84 \mathrm{Nm}$ each inning.

Static shoulder range of motion is also relevant due to the increased risk of injury noted with external rotation motion restriction. In a systematic review and metaanalysis of 15 studies, throwing athletes with preseason external rotation limitation (defined as throwing arm $<5^{\circ}$ greater than non-throwing arm) had an increased risk of injury with an odds ratio of $1.90 .^{39} \mathrm{It}$ should be noted that this study pooled all throwing athletes, and results may not be generalizable to just baseball athletes specifically. In a more focused metaanalysis of 3 studies investigating range of motion in baseball throwers, internal rotation and total range of motion were found to be significant predictors of injury but external rotation was not. ${ }^{40}$

While the associations between KPIs and injury risk have become increasingly clear to clinicians, a second question remains: are the KPIs measured in throwing analysis modifiable in a way that prevents injury? This represents a research gap in the literature ${ }^{1}$ and is an area that requires further research. Data thus far suggest that throwing mechanics are indeed modifiable. In a study of 46 pitchers with serial throwing assessments at an average of 12 months apart, $44 \%$ of flaws identified at the index assessment had been corrected. ${ }^{41}$ It remains to be shown whether this translates to injury prevention. Challenges in this pursuit include the 
Kinematically Guided Return to Sport Protocol with Video Motion Capture Analysis

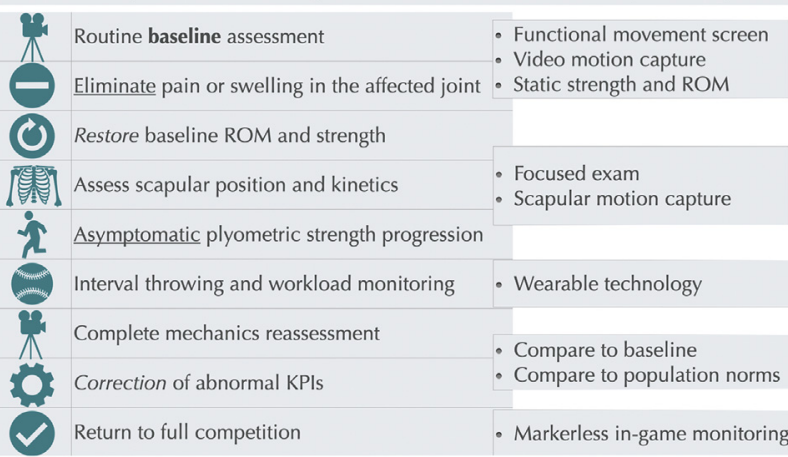

Fig 5. Return-to-sport protocol with kinematic assessments, video motion capture throwing analyses, and incorporation of next-generation technology (including wearable devices and marker-less motion capture). This protocol is adapted from Sgroi and Zajac ${ }^{42}$ with additions to reflect the role of biomechanics assessments and emerging technologies. (KPI, key performance indicators; ROM, range of motion.)

necessity of longitudinal throwing analysis data and injury reporting, the ethical limitations of a control group without modification of throwing pathomechanics, selection bias in pitchers presenting to throwing analysis centers and the multitude of confounding factors that contribute to athletic injuries.

\section{Return to Sport}

The role of throwing analysis in determining safe return to sport is another key area of interest that is under-represented in the literature to date. Sgroi and Zajac $^{42}$ proposed a return to throwing protocol after shoulder and elbow injury with a stepwise, criteriabased approach: (1) no pain or swelling in the affected joint, (2) restore baseline range of motion, (3) restore baseline strength, (4) assess for normal scapular position, (5) asymptomatic plyometric strength progression, (6) interval throwing and workload monitoring, (7) mechanics assessment, and (8) return to full competition. Biomechanical throwing analysis may have an important role in both the assessment of scapular position and throwing mechanics assessment, which are two steps along the return to sport cascade. Recognition of persistent pathomechanics (i.e., abnormal KPIs) at these stages has the potential to prevent reinjury, but this has yet to be shown in prospective studies. In addition, routine preinjury baseline throwing assessments can establish an athlete's true baseline for ROM, strength, and mechanics which can be used for comparison during the recovery protocol. Our recommended return to throwing protocol, adapted from Sgroi and Zajac, incorporates video motion capture throwing analysis, wearable technology, and marker-less in-game monitoring as outlined in Figure 5.

\section{Future Directions}

While throwing analysis still relies on video motion capture, which was performed as far back as the 1980s, the precision of measurement and complexity of analysis has continuously evolved. One area of recent progress has been the analysis of scapular mechanics. Scapular motion is challenging to accurately model with motion capture instruments. ${ }^{43}$ Increasing the number of calibration stages for scapular marker clusters placed on the acromion can improve accuracy, but this is not practical for all applications. ${ }^{44}$ A recent study sought to improve modeling of scapular kinematics using machine learning algorithms to better predict scapular motion based on 3D video motion capture. ${ }^{45}$ Scapular position predicted by the model was within $10^{\circ}$ of the positions measured by biplanar fluoroscopy. Further refinement of these models using similar computational techniques is an ongoing area of research.

Another topic of recent interest has been wearable technology for throwing analysis, which has the potential for more widespread use and could be incorporated into in-game throwing analysis. It also may have a role in guided rehabilitation particularly after ulnar collateral ligament reconstruction, as elbow varus torque can be monitored in real time. ${ }^{46}$ In a study using a wearable inertial device, elbow varus torque increased with long toss distance, a rehab variable that can be easily modified. ${ }^{47}$ One question with these devices has been the validity of their measurements. Camp et al. ${ }^{48}$ compared a wearable inertial measurement unit (MotusBASEBALL; Motus Global, Inc., Massapequa, $\mathrm{NY)}$ with gold-standard video motion capture in 10 varsity-level high school pitchers. While the wearable unit had good-to-excellent reliability with repeated measurements, significant differences between the gold standard and wearable unit were noted for arm slot, arm stress, and shoulder rotation. There was no difference in arm speed between the 2 measurement techniques. In a similar study, Boddy et al. ${ }^{49}$ used the same device and found strong correlations between the wearable technology and gold standard, but with significant differences in the magnitude of measurement for arm slot, arm speed, arm stress, and shoulder rotation. Taken together, these results suggest that there may be a role for wearable device measurements but that these measurements cannot be directly compared with video motion capture values reported in the literature. Further research is required to clarify the role of this device and others.

Of all measurements discussed so far, a common theme is some degree of wearable or marker-based attachment to a player's body to assist in measurements. A possible new frontier exists in marker-less tracking. Studies have begun to apply this technology for analysis of gait, $^{50-52}$ jumping, ${ }^{53}$ and baseball 
swinging. ${ }^{21}$ Application of marker-less technology to throwing has not been sufficiently validated to date, but research is ongoing, In a study comparing marker-based to marker-less motion capture during gait, differences between the 2 techniques were only $2.1 \mathrm{~cm}, 2.4 \mathrm{~cm}$, and $1.1 \mathrm{~cm}$ for segment locations at the shoulder, elbow, and wrist, respectively. ${ }^{50}$ In 2020 , Major League Baseball also introduced a motion capture system for in-game use. This system (Hawk-Eye Statcast; HawkEye Innovations Ltd., Basingstoke, UK) relies on 12 cameras positioned around an MLB ballpark. Five cameras are used for pitch tracking and operate at 100 frames per second, whereas 7 others are dedicated to player and batted ball tracking at 50 frames per second. ${ }^{54}$ Reported measurement capabilities include pitcher mechanics, release point, spin rate, spin axis, ball speed, and ball trajectory in addition to potential hitting and fielding-based metrics. Other systems with similar capabilities include KinaTrax (KinaTrax, Inc., Boca Raton, FL), SIMI (Simi Reality Motion Systems, Unterschleißheim, Germany), Theia3D (Theia Markerless, Inc., Kingston, Ontario, Canada), and DARI Motion (DARI Motion, Overland Park, KS). There is no peer-reviewed literature available to date to determine these systems' relative validity or injuryprevention capabilities, but the potential is exciting and future research is warranted.

\section{Conclusions}

Biomechanical analysis of the throwing athlete is most often performed with marker-based 3D video motion capture. This method allows for the noninvasive measurement of as many as 26 in vivo performance indicators. These measurements allow clinicians to identify pathomechanics that increase the risk of injury or poor performance. While video motion capture has remained the gold standard for throwing analysis for more than 3 decades, there have been recent advances in computational data analysis that may improve measurement precision. In addition, newer technologies are on the horizon including wearable devices and marker-less motion capture. It is important to rigorously validate these evolving technologies with the goal of widespread injury prevention for throwing athletes.

\section{References}

1. Bullock GS, Uhan J, Harriss EK, Arden NK, Filbay SR. The relationship between baseball participation and health: A systematic scoping review. J Orthop Sports Phys Ther 2020;50:55-66.

2. Posner M, Cameron KL, Wolf JM, Belmont PJ Jr, Owens BD. Epidemiology of Major League Baseball injuries. Am J Sports Med 2011;39:1676-1680.

3. Agel J, Rockwood T, Klossner D. Collegiate ACL injury rates across 15 sports. Clin J Sport Med 2016;26:518-523.
4. Gainor BJ, Piotrowski G, Puhl J, Allen WC, Hagen R. The throw: Biomechanics and acute injury. Am J Sports Med 1980;8:114-118.

5. Jobe FW, Tibone JE, Perry J, Moynes D. An EMG analysis of the shoulder in throwing and pitching. A preliminary report. Am J Sports Med 1983;1 1:3-5.

6. Jobe FW, Moynes DR, Tibone JE, Perry J. An EMG analysis of the shoulder in pitching. A second report. Am J Sports Med 1984;12:218-220.

7. Pappas AM, Zawacki RM, Sullivan TJ. Biomechanics of baseball pitching. A preliminary report. Am J Sports Med 1985;13:216-222.

8. Fleisig GS, Andrews JR, Dillman CJ, Escamilla RF. Kinetics of baseball pitching with implications about injury mechanisms. Am J Sports Med 1995;23:233-239.

9. Fleisig GS, Barrentine SW, Escamilla RF, Andrews JR. Biomechanics of overhand throwing with implications for injuries. Sports Med 1996;21:421-437.

10. Sisto DJ, Jobe FW, Moynes DR, Antonelli DJ. An electromyographic analysis of the elbow in pitching. Am J Sports Med 1987;15:260-263.

11. Weber AE, Kontaxis A, O'Brien SJ, Bedi A. The biomechanics of throwing: Simplified and cogent. Sports Med Arthrosc Rev 2014;22:72-79.

12. Fleisig G, Escamilla R, Andrews J, Matsuo T, Satterwhite Y, Barrentine S. Kinematic and kinetic comparison between baseball pitching and football passing. J Appl Biomech 1996;12:207-224.

13. Sgroi T, Chalmers PN, Riff AJ, et al. Predictors of throwing velocity in youth and adolescent pitchers. J Shoulder Elbow Surg 2015;24:1339-1345.

14. Tocci NX, Howell DR, Sugimoto D, Dawkins C, Whited A, Bae D. The effect of stride length and lateral pelvic tilt on elbow torque in youth baseball pitchers. J Appl Biomech 2017;33:339-346.

15. Manzi JE, Dowling B, Dines JS, et al. The association of stride length to ball velocity and elbow varus torque in professional pitchers [published online July 9, 2021]. $J$ Sports Sci https://doi.org/10.1080/02640414.2021. 1949190.

16. Dillman CJ, Fleisig GS, Andrews JR. Biomechanics of pitching with emphasis upon shoulder kinematics. J Orthop Sports Phys Ther 1993;18:402-408.

17. Moynes DR, Perry J, Antonelli DJ, Jobe FW. Electromyography and motion analysis of the upper extremity in sports. Phys Ther 1986;66:1905-1911.

18. Park SS, Loebenberg ML, Rokito AS, Zuckerman JD. The shoulder in baseball pitching: Biomechanics and related injuries-part 1. Bull Hosp Joint Dis 2002;61:68-79.

19. Fortenbaugh D, Fleisig GS, Andrews JR. Baseball pitching biomechanics in relation to injury risk and performance. Sports Health 2009;1:314-320.

20. Stokes H, Eaton K, Zheng NN. Shoulder external rotational properties during physical examination are associated with injury that requires surgery and shoulder joint loading during baseball pitching. Am J Sports Med 2021: 3635465211039850.

21. Sonnenfeld JJ, Crutchfield CR, Swindell HW, et al. An analysis of in vivo hip kinematics in elite baseball batters using a markerless motion-capture system. Arthrosc Sports Med Rehabil 2021;3:e909-e917. 
22. Kibler WB, Wilkes T, Sciascia A. Mechanics and pathomechanics in the overhead athlete. Clin Sports Med 2013;32:637-651.

23. Sciascia A, Thigpen C, Namdari S, Baldwin K. Kinetic chain abnormalities in the athletic shoulder. Sports Med Arthrosc Rev 2012;20:16-21.

24. Putnam CA. Sequential motions of body segments in striking and throwing skills: Descriptions and explanations. J Biomech 1993;26:125-135 (suppl 1).

25. Ellenbecker TS, Aoki R. Step by step guide to understanding the kinetic chain concept in the overhead athlete. Curr Rev Musculoskelet Med 2020;13:155-163.

26. Lintner D, Noonan TJ, Kibler WB. Injury patterns and biomechanics of the athlete's shoulder. Clin Sports Med 2008;27:527-551.

27. Chu SK, Jayabalan P, Kibler WB, Press J. The kinetic chain revisited: New concepts on throwing mechanics and injury. PM R 2016;8:S69-77.

28. Laudner K, Wong R, Evans D, Meister K. Lumbopelvic control and the development of upper extremity injury in professional baseball pitchers. Am J Sports Med 2021;49: 1059-1064.

29. Bullock GS, Strahm J, Hulburt TC, Beck EC, Waterman BR, Nicholson KF. The relationship of range of motion, hip shoulder separation, and pitching kinematics. Int J Sports Phys Ther 2020;15:1119-1128.

30. Digiovine NM, Jobe FW, Pink M, Perry J. An electromyographic analysis of the upper extremity in pitching. J Shoulder Elbow Surg 1992;1:15-25.

31. Escamilla RF, Andrews JR. Shoulder muscle recruitment patterns and related biomechanics during upper extremity sports. Sports Med 2009;39:569-590.

32. Townsend H, Jobe FW, Pink M, Perry J. Electromyographic analysis of the glenohumeral muscles during a baseball rehabilitation program. Am J Sports Med 1991;19: 264-272.

33. Chalmers PN, Wimmer MA, Verma NN, et al. The relationship between pitching mechanics and injury: A review of current concepts. Sports Health 2017;9:216-221.

34. Camp CL, Tubbs TG, Fleisig GS, et al. The relationship of throwing arm mechanics and elbow varus torque: Withinsubject variation for professional baseball pitchers across 82,000 throws. Am J Sports Med 2017;45:3030-3035.

35. Anz AW, Bushnell BD, Griffin LP, Noonan TJ, Torry MR, Hawkins RJ. Correlation of torque and elbow injury in professional baseball pitchers. Am J Sports Med 2010;38: 1368-1374.

36. Oyama S, Yu B, Blackburn JT, Padua DA, Li L, Myers JB. Improper trunk rotation sequence is associated with increased maximal shoulder external rotation angle and shoulder joint force in high school baseball pitchers. Am J Sports Med 2014;42:2089-2094.

37. Erickson BJ, Sgori T, Chalmers PN, et al. The impact of fatigue on baseball pitching mechanics in adolescent male pitchers. Arthroscopy 2016;32:762-771.

38. Okoroha KR, Meldau JE, Lizzio VA, et al. Effect of fatigue on medial elbow torque in baseball pitchers: A simulated game analysis. Am J Sports Med 2018;46:2509-2513.

39. Pozzi F, Plummer HA, Shanley E, et al. Preseason shoulder range of motion screening and in-season risk of shoulder and elbow injuries in overhead athletes: Systematic review and meta-analysis. Br J Sports Med 2020;54:1019-1027.

40. Bullock GS, Faherty MS, Ledbetter L, Thigpen CA, Sell TC. Shoulder range of motion and baseball arm injuries: A systematic review and meta-analysis. J Athl Train 2018;53:1190-1199.

41. Fleisig GS, Diffendaffer AZ, Ivey B, Aune KT. Do baseball pitchers improve mechanics after biomechanical evaluations? Sports Biomech 2018;17:314-321.

42. Sgroi TA, Zajac JM. Return to throwing after shoulder or elbow injury. Curr Rev Musculoskelet Med 2018;11:12-18.

43. Richardson RT, Rapp EA, Quinton RG, et al. Errors associated with utilizing prescribed scapular kinematics to estimate unconstrained, natural upper extremity motion in musculoskeletal modeling. J Appl Biomech 2017;33: 469-473.

44. Richardson RT, Nicholson KF, Rapp EA, Johnston TE, Richards JG. A comparison of acromion marker cluster calibration methods for estimating scapular kinematics during upper extremity ergometry. J Biomech 2016;49: 1255-1258.

45. Nicholson KF, Richardson RT, van Roden EAR, Quinton RG, Anzilotti KF, Richards JG. Machine learning algorithms for predicting scapular kinematics. Med Eng Phys 2019;65:39-45.

46. Bogdanov JM, Bolia IK, Benvegnu N, Michener LA, Weber AE, Petrigliano FA. Rehabilitation following ulnar collateral ligament reconstruction in overhead-throwing athletes. JBJS Rev 2021;9.

47. Dowling B, McNally MP, Laughlin WA, Onate JA. Changes in throwing arm mechanics at increased throwing distances during structured long-toss. Am J Sports Med 2018;46:3002-3006.

48. Camp CL, Loushin S, Nezlek S, Fiegen AP, Christoffer D, Kaufman K. Are wearable sensors valid and reliable for studying the baseball pitching motion? An independent comparison with marker-based motion capture. Am J Sports Med 2021:3635465211029017.

49. Boddy KJ, Marsh JA, Caravan A, Lindley KE, Scheffey JO, O'Connell ME. Exploring wearable sensors as an alternative to marker-based motion capture in the pitching delivery. PeerJ 2019;7:e6365.

50. Kanko RM, Laende EK, Davis EM, Selbie WS, Deluzio KJ. Concurrent assessment of gait kinematics using markerbased and markerless motion capture. J Biomech 2021;127:110665.

51. Kanko RM, Laende E, Selbie WS, Deluzio KJ. Inter-session repeatability of markerless motion capture gait kinematics. J Biomech 2021;121:110422.

52. Ota M, Tateuchi H, Hashiguchi T, Ichihashi N. Verification of validity of gait analysis systems during treadmill walking and running using human pose tracking algorithm. Gait Posture 2021;85:290-297.

53. Drazan JF, Phillips WT, Seethapathi N, Hullfish TJ, Baxter JR. Moving outside the lab: Markerless motion capture accurately quantifies sagittal plane kinematics during the vertical jump. J Biomech 2021;125:110547.

54. Jedlovec B. Introducing Statcast 2020: Hawk-Eye and Google Cloud. MLB Technology Blog Vol 20212020. 